\title{
Cómputo Ubicuo en el Control Canino para Actividades de Caza
}

\author{
Adrian Castañeda, Pedro Damián, María Andrade \\ Universidad de Colima, Facultad de Telemática \\ \{acasta66, damian, mandrad\}@ucol.mx
}

\begin{abstract}
Resumen. En este trabajo se expone una investigación donde se analizan varios de los principales trabajos en Interacción Animal Computadora (ACI) y los distintos dispositivos tecnológicos en comunicación que existen en la actualidad para animales. Estos dispositivos son usados por el ser humano para interactuar con los animales, lo cual nos permitirá diseñar y desarrollar una herramienta para telefonía celular. El software será enfocado principalmente para saber la localización, establecer comunicación y conocer el estado físico de perros de trabajo cuando realizan una actividad, todo ello con el propósito de tener una mejor interacción y control sobre estos animales.
\end{abstract}

Palabras Clave: Interacción Animal-Computadora, Perro, Caza, Telefonía Celular.

\section{Introducción}

Actualmente el uso de las tecnologías de información se encuentra presente en las diferentes actividades de la sociedad. El ser humano puede interactuar de forma transparente con objetos de uso diario, que cuentan con capacidades computacionales que les permiten percibir y capturar el mundo que rodea al usuario, con la finalidad de adaptar su comportamiento y proporcionar un servicio en el lugar y el momento necesario.

Hoy en día la tecnología se ha ampliado ofreciendo aplicaciones para los dueños de animales, con el objetivo de mejorar la calidad de vida de éste, además de proporcionar los estímulos que necesita y profundizar en las necesidades de la mascota, que en este estudio se enfoca a los perros.

Los dispositivos tecnológicos para animales que existen en la actualidad, apoyan la interacción con sus dueños, y frecuentemente se observan perros de trabajo en diferentes actividades de ayuda al hombre, como lo es en la cacería. Para apoyar de mejor manera esta relación se propone desarrollar una herramienta que permita la interacción mediante la utilización de telefonía celular. 


\section{Antecedentes}

El origen de la caza ha estado presente a lo largo de la historia y la actividad de perseguir, atrapar o matar cualquier animal, comúnmente es realizada por humanos como alimento, comercio y recreación [10].

Desde su domesticación el perro ha acompañado y apoyado al cazador en sus actividades, por lo tanto el hombre ha ido seleccionando al perro para distintas tareas, de tal forma que en la actualidad existen distintas razas especializadas, con diferentes características de instinto, fuerza, olfato, ladrido, valentía, iniciativa y tamaño [9]. Asimismo, la clasificación de la Federación Cinológica Internacional (FCI) seis de sus diez grupos de razas de perros son de caza, desde perros sabuesos, lebreles, terriers, perros de muestra, perros cobradores, perros de agua, perros levantadores y perros nórdicos [16].

Por otra parte en 1992 la empresa IBM y BellSouth creó el teléfono móvil Simon Personal Communicator, adelantado para su época, combinando las características de teléfono móvil, localizador, agenda electrónica y pantalla táctil, con aplicaciones de calendario, libreta de direcciones, correo electrónico y juegos [8].

Además, hoy en día es muy usado el teléfono inteligente, el cual es un teléfono móvil convencional con capacidad de almacenar datos y realizar actividades semejantes a una computadora. Es llamado inteligente por ser usado como procesador de bolsillo. Generalmente tienen pantalla táctil, acceso a correo electrónico, y permiten la instalación de programas, multitarea, acceso a internet, funciones multimedia y manejo de múltiples formatos de documentos [15].

No obstante, uno de los pioneros en interfaz humano-animal fue Resner (2001), quien diseñó un sistema remoto de interacción humano-perro llamándolo Rover@Home. El objetivo fue construir un dispositivo que permitiera a los animales y a sus propietarios tener una interacción mientras se encuentran separados geográficamente, de la misma manera que un teléfono permite a dos seres humanos distantes comunicarse, desarrollaron un dispositivo que realiza una función similar entre animales domésticos y seres humanos. Este sistema permite enseñar a sus perros nuevas tareas, usando internet, una computadora y técnicas de entrenamiento, aplicando principios de diseño de Interacción Humano Computadora (IHC). En su investigación, explica que hay una serie de razones de por qué los perros representan un nuevo e interesante reto de diseño. En primer lugar, que los perros son criaturas muy sociables y por lo tanto se benefician de una interacción bien diseñada con un compañero remoto humano. En segundo lugar, los humanos están familiarizados con los perros y esa interacción proporciona un rico conjunto de conocimiento que sirve de guía para el desarrollo. Y tercero, menciona que el proceso de pensar a través de lo que es una interacción bien diseñada en el contexto de una computadora entre un perro y un ser humano aclara nuestra comprensión para la creación de dispositivos para humanos y animales por igual. Argumenta que los puntos clave de diseño centrado en el usuario son también muy útiles guiando el proceso de diseño de dispositivos para animales [1].

Adicional a lo anterior, años más tarde Demi Mankoff (2005), desarrolló un sistema de vigilancia a distancia para la interacción humano-perro, ayudando a los perros a mantener un sentido de conexión con sus dueños mediante un juego de lanzarle pelotas de tenis al perro, mitigando el aislamiento social, ya que los humanos se 
encuentran muchas horas del día fuera de casa o trabajando. El sistema consiste en una Interfaz Usuario-Humano (HUI) y una Interfaz Usuario-Perro (DUI). Demostraron que los sonidos y videos son más efectivos en el interés del perro. El sistema provee a los seres humanos herramientas para proporcionar a los animales una mejor calidad de vida $[2,3]$.

\section{Tecnología de Comunicación, Rastreo e Interacción con Animales}

Actualmente, en el Instituto de Georgia de Tecnología están trabajando en un proyecto llamado FIDO (Facilitating Interactions for Dogs with Occupations), el cual investiga aspectos fundamentales en la tecnología portátil para apoyar la comunicación entre perros de trabajo y sus dueños. Utiliza un chaleco para perros con diferentes sensores (morder, tirar y gestos de nariz) y muestra la creación de sensores portátiles que los perros pueden activar. El principal objetivo de este proyecto es investigar los aspectos fundamentales de tecnología portátil de apoyo en la comunicación de los perros de trabajo a sus dueños. Los perros proporcionan información a través de los sensores integrados en el chaleco del perro, creando interfaces de usuario para perro, los cuales fueron probados con tres diferentes perros de asistencia entrenados para evaluar la facilidad de la interacción. A partir de un estudio piloto, determinaron qué tipo de sensores para perro puede ser más apto para favorecer la interacción. Con la colocación de múltiples sensores en el chaleco del perro para comunicar una variedad de mensajes, probaron cada sensor individualmente en la misma ubicación del lado izquierdo del chaleco del perro, y para cada sensor midieron las lecturas mediante una serie de interacciones con el perro. Los resultados del estudio piloto fueron extremadamente alentadores, y demostraron que es posible crear sensores portátiles que los perros pueden activar para comunicarse con sus dueños. Mencionan que existe una gran cantidad de trabajo que aún no se ha hecho, faltando probar los sensores en ambos lados del chaleco para determinar qué ubicación es alcanzable para diferentes tipos de cuerpos de perro y determinar el área óptima de cada tipo de sensor. Necesitan descubrir la colocación de los sensores para entrenar los perros con los diferentes sensores en sus cuerpos. Esta tecnología podría ser fácilmente adaptada a otras profesiones caninas como perros policía o perros militares, proporcionando a los perros la habilidad de comunicarse con sus dueños claramente [4].

La tecnología de la comunicación ha favorecido la conexión entre las personas y ha sido una parte integral de nuestra vida cotidiana. La tecnología móvil se ha usado por un par de décadas para la comunicación entre personas y recientemente para monitorear eventos remotos, salud y actividad, estando presente en todos los sectores de la vida. Recientemente se ha ampliado para ofrecer aplicaciones para los dueños de perros, y se ha investigado el uso de la tecnología para la interacción entre personas y perros, ya que un perro es una parte esencial de la vida cotidiana de su dueño, por ser significativa la inversión en tiempo y esfuerzo en ellos. Además, la tecnología está encontrando lentamente el negocio en los perros, ya que el desarrollo de tecnología relacionada con mascotas es aún un campo nuevo y recientemente investigaciones en 
tecnología desarrollada para animales se ha concentrado en dos áreas: una que interactúa con los animales en forma remota [1] y el monitoreo del cuidado o bienestar de éste [17]. Ambos aspectos tienen el mismo objetivo, mejorar la calidad de vida del animal proporcionándole los estímulos que necesita o profundizar en el conocimiento del dueño en la mascota. Como la tecnología de comunicación para el monitoreo de perros es relativamente nueva, en casos específicos de diseños con nuevos prototipos o con aplicaciones existentes son dirigidas a grupos específicos de usuarios que por lo general son cazadores [5].

Asimismo, los animales cada vez están más integrados en contextos interactivos de tecnologías digitales, el presente y futuro de dichas tecnologías es un tema relevante para la investigación de HCI. Un campo de estudio en actividades de caza es el uso de dispositivos de rastreo GPS en perros. Siendo la interacción entre perros y seres humanos afectada con nuevas tecnologías, además esta interacción humano animal es apoyada con la tecnología digital, como los dispositivos para entrenar perros utilizando descargas eléctricas, cuidado de perros con control remoto de dispositivos de alimentación, así como perros de vigilancia por medio de localización por GPS. Sin embargo, la investigación en esta área es limitada por lo que es difícil entender la experiencia de esas nuevas tecnologías. Los cazadores utilizan dispositivos GPS para monitorear los perros cambiando la relación entre perros y humanos cuando se introduce nueva tecnología. El GPS permite a los cazadores obtener una comprensión mejor de lo que el perro está haciendo para apoyarlo en sus acciones. El perro proporciona información al cazador de donde está la presa, al usar los dispositivos GPS se tiene una nueva forma de caza y visión a distancia. Se podría interpretar que esta nueva forma le quita placer a la caza, sin embargo, añade una nueva dimensión pero no le quita el reto de la caza [6].

Por otra parte, el uso de la tecnología en rastreo en animales está en aumento. Sin embargo es poco investigada restringiéndose a perros y gatos. Los dueños pueden escoger entre una gran variedad de diseños de dispositivos GPS [7]. En investigaciones de interacción humano-animal, pocos estudios muestran el uso de dispositivos GPS durante actividades como la cacería [5]. Sin embargo, la tecnología de rastreo ha sido usada con animales por un largo tiempo, por ejemplo para monitorear animales salvajes en peligro de extinción en esfuerzos de conservación, o coordinar equipos de trabajo en actividades durante la cacería, ofreciendo nuevas oportunidades de interacción [7].

Actualmente existe en el mercado para la interacción con animales una amplia selección de productos de tecnología de comunicación, algunos sistemas de comunicación basados en Internet, por ejemplo iSeePet Webcam/Feeder en donde los usuarios pueden ver la actividad de sus mascotas y darles de comer en forma remota [18]. Webcams son utilizadas para el monitoreo de mascotas [19]. Es decir, existe mucha tecnología relacionada con el rastreo [20].

Los sistemas de rastreo en el mercado se basan principalmente en GPS, donde los dispositivos usados en cacería contienen un transmisor GPS para el perro y un dispositivo GPS de mano para el usuario. La unidad GPS está unida al collar o arnés del perro durante el rastreo. En los sistemas basados en Sistema global móvil (GSM), un teléfono móvil se utiliza como dispositivo de mano. Los sistemas basados en GSM requieren estar conectados con un proveedor de servicio de Internet. Sin embargo, los sistemas basados en frecuencia muy alta (VHF) tienen un rango máximo de 3 a $10 \mathrm{~km}$ 
dependiendo del entorno. En contraparte, en los sistemas basados en GSM no hay un rango límite siempre y cuando la red GSM esté disponible para ambas unidades. Hay sistemas GPS que sólo utilizan señal de radio frecuencia para enviar la ubicación al receptor de mano y saber qué tan lejos está el perro [5]. El sistema VHF no tiene un rango de operación muy amplio en comparación con sistemas basados en GSM el cual es, sin duda, el más fiable y más fácil de utilizar.

\section{Trascendencia de la Propuesta}

Actualmente es común ver perros de trabajo en actividades de apoyo a personas con capacidades diferentes. Tal como la organización de compañeros caninos para la independencia, la cual es sin fines de lucro para mejorar la vida de personas con discapacidad, proporcionando perros de asistencia altamente capacitados [11]. En seguridad como perros policía, son utilizados por uno de los grupos especiales del ayuntamiento de Chihuahua el cual ya tiene 10 años y utilizan perros de la raza pastor belga millinois [12]. En la unidad canina de la policía federal de México también utilizan perros para la revisión de personas, mercancías y objetos [13]. En búsqueda y rescate en asociaciones civiles como la brigada canina de la Universidad de Colima que ha apoyado en búsqueda de personas extraviadas, sepultadas en escombros así como ahogadas [14], y en actividades de caza empleando diferentes tipos de razas de perro.

Por otra parte, hoy en día es frecuente ver anuncios de perros extraviados en diarios, revistas, anuncios en paredes y postes en la calle, así como en páginas de internet. Un estudio realizado en España en el 2010 por la fundación Affinity sobre el abandono de animales de compañía, destaca que uno de los motivos de abandono es el fin de temporada de caza y entre las medidas para erradicar el abandono es tener un mayor control sobre la caza con animales [21].

Además, la Organización Panamericana de la Salud cambió el título de perro callejero a "perro de dueño irresponsable", ya que la gran mayoría de los callejeros llegan a la vía pública debido a que sus propietarios ya no los quieren en sus casas porque dejan de considerarlos graciosos o tiernos, o porque no pueden asumir los gastos que implica el cuidado del animal. Algunos animales llegan a la calle por extravío. Según datos de la Secretaría de Salud capitalina, cada año 18 mil perros se pierden o son abandonados por sus dueños. Durante 2011, en los dos centros de control canino a cargo de esta secretaría se recibieron aproximadamente 60 mil perros y gatos callejeros, de los cuales 9 de cada 10 son "sacrificados" debido a que no fueron reclamados o no tuvieron la fortuna de ser adoptados. La tenencia responsable implica entender que tener un animal es una gran responsabilidad, como tener un hijo; "es un miembro más en la familia, al igual que todos necesita nutrición, cuidados, atenciones, recreación, amor y protección" [22]. Con respecto a los porcentajes de animales abandonados o que se pueden localizar en las ciudades, es difícil calcularlos pues no hay reportes documentados de estos eventos en ningún medio.

También, al realizar algunas actividades de caza se utilizan perros para rastrear, localizar, perseguir, apuntar y recoger presas [9], donde los perros pueden desplazarse largas distancias perdiéndose de la vista de sus dueños, sin poder escuchar sus 
ladridos, lo que origina que en algunas ocasiones los perros se extravíen. Por lo tanto, en ocasiones es imposible que el cazador pueda dar instrucciones a los perros cuando se encuentran retirados haciendo el menor ruido posible, así como conocer el estado físico del perro.

De ahí que, la práctica de la cacería puede ser apoyada con la utilización de telefonía celular mediante el desarrollo de una aplicación, la cual apoyaría al cazador en todo momento en conocer la ubicación y ruta trazada por el perro, así como poder trasmitirle alguna orden al perro, siempre con el conocimiento de su estado físico durante la actividad y precisamente de esta necesidad se origina esta propuesta de investigación. En la figura 1, se muestra uno de los escenarios diseñados, utilizando telefonía celular para tener un mejor control sobre los perros, de tal manera se adapta al chaleco del perro un compartimento para portar un celular, el cual transmite el ritmo cardiaco y su ubicación, además recibe instrucciones de su dueño, proporcionando al cazador información útil para tomar decisiones mientras se realiza la actividad de caza.

Se tiene el propósito de que esta herramienta pueda ser utilizada por asociaciones de clubes cinegéticos y búsqueda y rescate, no sin antes ser evaluada.

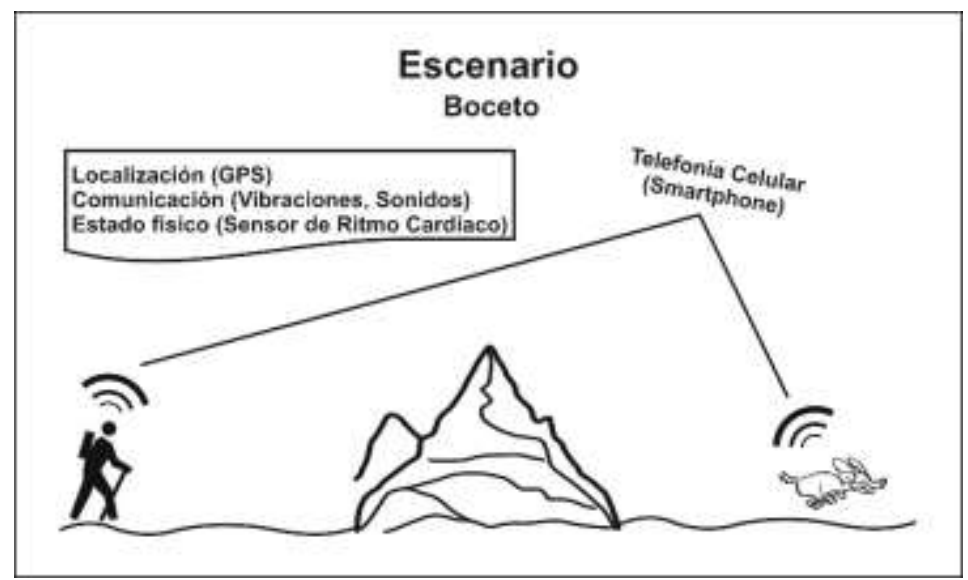

Figura 1. Escenario de la práctica de cacería con apoyo de la tecnología.

Para la actividad de evaluación se tiene preparado un instrumento de valoración y análisis con expertos.

\section{Trabajo en Desarrollo}

Actualmente se está desarrollando la herramienta de apoyo para perros de trabajo que permite la interacción entre el perro y su dueño. Se registra el ritmo cardiaco del perro, su ubicación así como el registro de las instrucciones. Con ello, es posible la evaluación de la aplicación tecnológica. Por estas razones, se decidió investigar y documentar la implementación de cómputo ubicuo en el control canino mediante el 
desarrollo de una aplicación para telefonía celular (Smartphone) en las actividades de caza.

En la figura 2 se muestra la forma de interacción entre los dispositivos en la que el sensor transmite el ritmo cardiaco mediante comunicación Bluetooth al Smartphone que están colocados en el chaleco del perro.

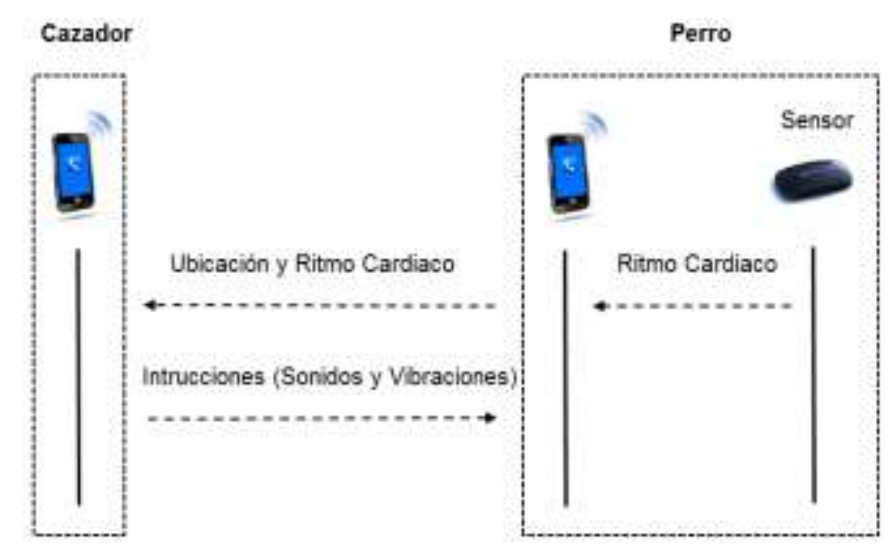

Figura 2. Comunicación entre los diferentes dispositivos.

A su vez, el Smartphone transmite su ubicación y el ritmo cardiaco a través de servicio de datos de telefonía celular al Smartphone del cazador, el cual envía instrucciones por medio de sonidos y vibraciones al Smartphone que tiene el perro.

\section{Conclusiones}

El cómputo ubicuo ha demostrado ser una opción muy importante en varias actividades de la vida cotidiana del ser humano. Así mismo se ha extendido para apoyar en distintas acciones para beneficio de los animales debido a un aumento de dispositivos tecnológicos que favorece la interacción de los dueños con sus mascotas, fortaleciendo la relación del hombre con los animales en sus necesidades. Por lo tanto, mediante el desarrollo de una aplicación para telefonía celular (Smartphone), consideramos que su implementación y uso puede favorecer las actividades de caza. Esto se llevará a cabo con la utilización de sonidos, vibraciones, localización y ritmo cardiaco. La herramienta se probará y evaluará para tener un mejor control del perro.

\section{Referencias}

1 Resner, B.I., Rover@Home: Computer Mediated Remote Interaction for Dogs. Media Arts and Sciences. MS, Cambridge, Massachusetts Institute of Technology. 2001 
2 Mankoff, D., Dey, A. K., Mankoff, J. \& Mankoff, K. (2005). Supporting interspecies social awareness: using peripheral displays for distributed pack awareness. In P. Baudisch, M. Czerwinski \& D. R. Olsen (eds.), UIST (p./pp. 253-258): ACM. ISBN: 1-59593-271-2

3 Mancini, Clara (2013). Animal-Computer Interaction (ACI): changing perspective on HCI, participation and sustainability. In: CHI 2013, 27 April-02 May 2013, Paris, pp. 2227-2236.

4 Jackson, M. M., Zeagler, C., Valentin, G., Martin, A., Martin, V., Delawalla, A., Blount, W., Eiring, S., Hollis, R., Kshirsagar, Y. \& Starner, T. (2013). FIDO - facilitating interactions for dogs with occupations: wearable dog-activated interfaces. In K. V. Laerhoven, D. Roggen, D. Gatica-Perez \& M. Fukumoto (eds.), ISWC (p./pp. 81-88), ACM. ISBN: 978-14503-2127-3

5 Paldanius, M., Kärkkäinen, T., Väänänen-Vainio-Mattila, K., Juhlin, O. \& Häkkilä, J. (2011). Communication technology for human-dog interaction: exploration of dog owners' experiences and expectations. In D. S. Tan, S. Amershi, B. Begole, W. A. Kellogg \& M. Tungare (eds.), CHI (p./pp. 2641-2650), ACM. ISBN: 978-1-4503-0228-9

6 Weilenmann, A. \& Juhlin, O. (2011). Understanding people and animals: the use of a positioning system in ordinary human-canine interaction. In D. S. Tan, S. Amershi, B. Begole, W. A. Kellogg \& M. Tungare (eds.), CHI (p./pp. 2631-2640), ACM. ISBN: 978-14503-0228-9

7 Mancini, C., Van der Linder, J., Bryan, J., Stuart, A. Exploring Interspecies Sensemaking: Dog Tracking Semiotics and Multispecies Ethnography.UbiComp 12, Sep 5-Sep 8, 2012, Pittsburgh, USA. Copyright 2012 ACM 978-1-4503-1224-0/12/09

8 Corcoran P. (2011). "A Bitter Pill or a Better Tablet? A historical perspective on tablet computers" Available: http://theinstitute.ieee.org/technology-focus/technology-history/abitter-pill-or-a-better-tablet

9 Gutierrez D. Hunting Dog Information. Recuperado el 18 de junio de 2014, de http://dogcare.dailypuppy.com/hunting-dog-information-3933.html

10 El Diccionario de la lengua española (DRAE), http://buscon.rae.es/drae/srv/search?id= 3stRVstbFDXX2aGfL8D9

11 Canine Companions for Independence, http://www.cci.org/site/c.cdKGIRNqEmG/b.3978475/k.3F1C/Canine_Companions_for_Ind ependence.htm

12 Ayuntamiento http://www.municipiochihuahua.gob.mx/policia/dependencia/grupos_especiales.aspx

13 Comisión Nacional de Seguridad, http://www.cns.gob.mx/portalWebApp/ShowBinary?nodeId=/BEA+Repository/1332058//ar chivo

14 Universidad de Colima, http://www.ucol.mx/ssocial/bcanino/antecedentes.htm

15 John D. Sutter. (2010). ¿Qué es y qué debo esperar de un "teléfono inteligente"?. Recuperado el 10 de junio de 2014, de http://mexico.cnn.com/tecnologia/2010/10/21/que-esy-que-debo-esperar-de-un-telefono-inteligente

16 Gutiérrez, J. (2012). Lista Oficial de Razas Caninas de la FCI. Recuperado el 18 de junio de 2014, de http://www.adiestradorcanino.com/webdelperro/lista-oficial-de-razas-caninas-dela-fci/84

17 Yonezawa, K., Miyaki, T. \& Rekimoto, J. (2009). Cat@Log: sensing device attachable to pet cats for supporting human-pet interaction. Advances in Computer Entertainment Technology (p./pp. 149-156), ACM. ISBN: 978-1-60558-864-3

18 Grundner A. (2004). iSeePet Webcam/Feeder. Recuperado el 16 de junio de 2014, de http://www.ehomeupgrade.com/2004/04/24/iseepet-webcamfeeder/

19 Fernández A. (2013). Webcams para ver animales en directo. Recuperado el 8 de junio de 2014, de http://www.consumer.es/web/es/medio_ambiente/naturaleza/2013/03/ 28/216266.php 
20 Tobar E. (2014). 3 gadgets para el monitoreo y cuidado de tu mascota. Recuperado el 18 de junio de 2014, de http://www.maestrosdelweb.com/editorial/tecnologia-usable-gadgetsmascota/

21 Casamayor P. Responsable del proyecto. (2010). Estudio Fundación Affinity sobre el abandono de animales de compañía. Recuperado el 18 de junio de 2014, de http://www.fundacion-affinity.org/sites/default/files/EstudioAbandono2010.pdf

22 Morán L. (2012). Proponen solución al problema de los perros callejeros. Recuperado el 18 de junio de 2014, de http://ciencia.unam.mx/leer/109/Proponen_solucion_al_problema_ de_los_perros_callejeros 